\title{
Rigidity analysis of protein biological assemblies and periodic crystal structures
}

\author{
Filip Jagodzinski ${ }^{3}$, Pamela Clark', Jessica Grant ${ }^{2}$, Tiffany Liư ${ }^{4}$, Samantha Monastra', Ileana Streinu ${ }^{3,4^{*}}$ \\ From Second IEEE International Conference on Computational Advances in Bio and Medical Sciences \\ (ICCABS 2012) \\ Las Vegas, NV, USA. 23-25 February 2012
}

\begin{abstract}
Background: We initiate in silico rigidity-theoretical studies of biological assemblies and small crystals for protein structures. The goal is to determine if, and how, the interactions among neighboring cells and subchains affect the flexibility of a molecule in its crystallized state. We use experimental $X$-ray crystallography data from the Protein Data Bank (PDB). The analysis relies on an effcient graph-based algorithm. Computational experiments were performed using new protein rigidity analysis tools available in the new release of our KINARI-Web server http:// kinari.cs.umass.edu.
\end{abstract}

Results: We provide two types of results: on biological assemblies and on crystals. We found that when only isolated subchains are considered, structural and functional information may be missed. Indeed, the rigidity of biological assemblies is sometimes dependent on the count and placement of hydrogen bonds and other interactions among the individual subchains of the biological unit. Similarly, the rigidity of small crystals may be affected by the interactions between atoms belonging to different unit cells.

We have analyzed a dataset of approximately 300 proteins, from which we generated 982 crystals (some of which are biological assemblies). We identified two types of behaviors. (a) Some crystals and/or biological assemblies will aggregate into rigid bodies that span multiple unit cells/asymmetric units. Some of them create substantially larger rigid cluster in the crystal/biological assembly form, while in other cases, the aggregation has a smaller effect just at the interface between the units. (b) In other cases, the rigidity properties of the asymmetric units are retained, because the rigid bodies did not combine.

We also identified two interesting cases where rigidity analysis may be correlated with the functional behavior of the protein. This type of information, identified here for the first time, depends critically on the ability to create crystals and biological assemblies, and would not have been observed only from the asymmetric unit. For the Ribonuclease A protein (PDB file 5RSA), which is functionally active in the crystallized form, we found that the individual protein and its crystal form retain the flexibility parameters between the two states. In contrast, a derivative of Ribonuclease A (PDB file 9RSA), has no functional activity, and the protein in both the asymmetric and crystalline forms, is very rigid.

For the vaccinia virus D13 scaffolding protein (PDB file 3SAQ), which has two biological assemblies, we observed a striking asymmetry in the rigidity cluster decomposition of one of them, which seems implausible, given its symmetry. Upon careful investigation, we tracked the cause to a placement decision by the Reduce software concerning the hydrogen atoms, thus affecting the distribution of certain hydrogen bonds. The surprising result is that the presence or lack of a very few, but critical, hydrogen bonds, can drastically affect the rigid cluster decomposition of the biological assembly.

\footnotetext{
* Correspondence: istreinu@smith.edu

${ }^{3}$ Department of Computer Science, Central Washington University, Ellensburg, WA, USA

Full list of author information is available at the end of the article
} 
Conclusion: The rigidity analysis of a single asymmetric unit may not accurately reflect the protein's behavior in the tightly packed crystal environment. Using our KINARI software, we demonstrated that additional functional and rigidity information can be gained by analyzing a protein's biological assembly and/or crystal structure. However, performing a larger scale study would be computationally expensive (due to the size of the molecules involved). Overcoming this limitation will require novel mathematical and computational extensions to our software.

\section{Background}

Proteins are essential parts of all organisms. Some have structural roles, some help to mitigate enzymatic reactions; they are involved in virtually all cellular processes. Proteins are made up of long chains of amino acids joined end-to-end, whose interactions cause the chain to fold forming additional secondary, tertiary, and quaternary structures [1]. The function of a protein is often correlated with conformational changes that are driven by molecular interactions. To better understand a protein's function, its motion can be simulated and critical chemical interactions among its constituent parts can be observed. However, such simulations are computationally intense and often intractable. An alternative method is to model a protein as a mechanical structure made up of rigid and flexible parts. The overall rigidity of such a structure can be analyzed using an effcient combinatorial algorithm, which is described below, and the resulting decomposition into rigid clusters yields insights into the possible motions of the protein. Rigidity analysis has proven its usefulness [2] in giving insights about protein flexibility, but until now such studies have been performed only on a small set of proteins, each one taken in isolation.

Over $87 \%$ of the protein structures deposited in the Protein Data Bank have been solved with X-ray crystallography. Also, protein function is correlated with flexibility, and one would expect that in tightly packed crystals, some flexibility would also be lost. Indeed, in work done by Zhang, et. al [3], an analysis of 25 crystal forms of T4 lysozyme revealed that crystal contacts perturb a protein's backbone by 0.2 to $0.5 \AA$. Protein flexibility studies using rigidity analysis have been performed until now primarily on individual asymmetric units (the smallest part of a protein that is needed to recreate the protein's biological functional form) from the data available in the PDB.

For some proteins the asymmetric unit is identical to the biological assembly. However, for many proteins, especially those determined via X-ray crystallography, the asymmetric unit is different from the biological assembly. Depending on how the protein was crystallized, the relationship between the asymmetric unit and the biological assembly can vary from protein to protein. Some biological assemblies are composed of many copies of the asymmetric unit.
The PDB file contains only the atomic coordinates of the asymmetric unit, and it is natural to expect that its rigidity analysis may not always reflect the flexibility properties of the biological form. One extreme example is a viral capsid: the icosahedral type is composed of 60 repeating units, and each one may contain several monomeric units. To gain information on the flexibility of the virus would require building the entire assembly, but so far, no existing software automatically performs this task.

In this paper, we demonstrate that new insights into protein flexibility can be obtained by performing rigidity analysis on biological assemblies and protein crystal structures. These computational experiments were performed using an in-house implementation and new tools now made available in the new release of our KINARI-Web server http://kinari.cs.umass.edu, which relies on an effcient graph-based algorithm. For a proofof-concept, we incorporated some freely available scripts for building crystals. KINARI [4] comes with tools for curating the input data and for visualizing the rigidity results. To investigate the rigidity of biological assemblies, we developed and integrated with KINARI a BioAssembly tool, that permits a user to generate biological structures from the asymmetric units typically recorded in PDB files. Figure 1 (right) shows its output of rigid clusters for a small crystal structure of protein 2ON8; different colors indicate different rigid bodies.

We tested our method on 982 crystals of various sizes, build from 324 protein structure files retrieved from the $\mathrm{PDB}$. We found that the rigidity results vary among different proteins, an indication that there is additional information to be extracted from rigidity analysis of crystals and biological assemblies, as opposed to just a single asymmetric unit. In some cases, the biological unit, analyzed in isolation, exhibits significantly more flexibility than its crystal counterpart, while in others, the rigidity properties appear to be stable in the two forms.

For these 324 proteins, we build the unit cell, as well as $2 \times 1 \times 1$, and $2 \times 2 \times 1$ crystals from the asymmetric unit data in each PDB file. The rigidity results were analyzed to reveal trends in the rigidity properties of the crystal lattices. This indicated that chemical interactions among individual proteins in a lattice can greatly impact the crystallized form of the protein. However, some crystal 

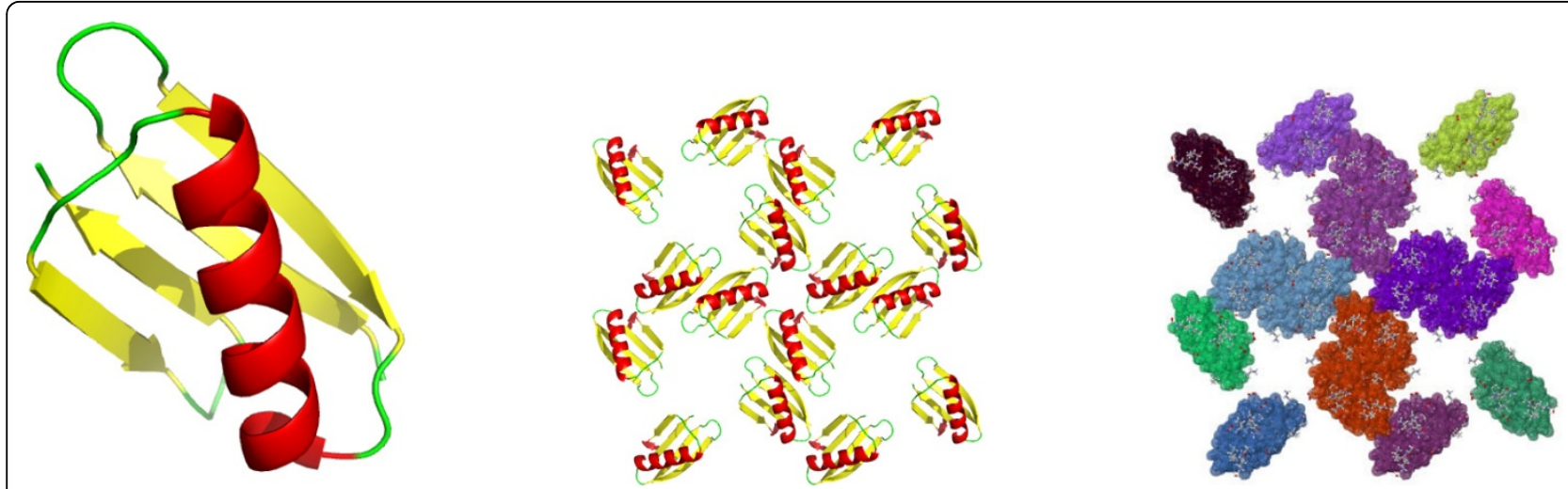

Figure 1 (left) The cartoon rendering of the asymmetric unit of PDB file 20N8 [29]; (center) a small crystal structure for 20N8, and (right) its flexibility analysis results obtained by using the KINARI software. The different colors designate distinct rigid clusters.

structures are not stabilized by these additional chemical interactions, and this may reflect a biologically relevant property. Indeed, it is reported in the literature [5] that the Ribonuclease A (RNase A) protein (PDB code 5RSA) retains its function even in the crystallized form used for its X-Ray structure determination. On the other hand, a derivative of RNase A protein (PDB code 9RSA) retains only $1 \%$ of its activity, and our analysis revealed that it is very rigid, in both the asymmetric and crystal lattice forms. The rigidity results of these two forms of RNase A correlate surprisingly accurately with their reported activities.

We describe now the theoretical foundations of our method, the computational experiments, detailed case studies for several crystal structures and biological assemblies, and our survey of the rigidity properties of 982 crystal. We conclude with a discussion of future work.

\section{Rigidity analysis and mechanical models of proteins}

Geometric and combinatorial methods from rigidity theory have been applied to the study of protein flexibility, by associating a network of nodes (atoms) connected by rigid bars (bonds and other stabilizing interactions). The study of rigidity and flexibility of these bar-and-joint frameworks dates back to a counting rule identified in 1864 by J.C. Maxwell [6]. An extension to 3-dimensional structures known as body-bar and body-hinge frameworks has been proven by Tay [7], and led to effcient algorithms [8,9] for rigidity analysis. This technique has been applied to understanding flexibility of glasses, proteins [2] and other molecular structures.

In a molecule modeled as a body-bar-hinge framework, a body is a set of atoms rigidly attached to each other. For example, methane (Figure 2, two left subfigures) is rigid, because all the pair-wise distances between the atoms in this small molecule are determined by the existing covalent bond length and angle constraints. Ethane (Figure 2, center) exhibits one degree of flexibility, because the $\mathrm{C}-\mathrm{C}$ bond permits rotation. Rotatable bonds are modeled as hinges. Two peptide units on a protein backbone (Figure 2, two right subfigures) lead to three rigid bodies, connected by two hinges.

\section{Rigidity analysis of protein structures}

As methods for analyzing protein rigidity have advanced, rigidity analysis has been used successfully to investigate and study structural and functional aspects of various molecules. It has been found that the active sites of enzymes tend to be more rigid than other regions [10]. Rader et al. [11] have measured the increase in flexibility in proteins after thermal denaturation; they proposed that the folding of proteins into their three-dimensional structure be seen as a process of increasing rigidity, and verified it for the protein Rhodopsin. Protein rigidity analysis has been primarily applied to single protein units, although biomolecules and larger macromolecular complexes have been the focus of at least one recent study [12]. However, larger crystal structures have not been the subject of rigidity analysis prior to this work.

\section{The protein data bank, biomolecules and crystals}

The proteins used in this study were retrieved from the $\mathrm{PDB}$, a repository for experimental protein structure data with over 82,000 entries at this time. A PDB file contains atom coordinates for the asymmetric unit of a protein, i.e. the minimal set of atoms necessary to reproduce the complete protein biological assembly and crystal which was analyzed with X-ray diffraction. A PDB file has information on how to create the biomolecule, the functional biological unit, and unit cell, the repeating unit of the crystal. A unit cell vector is a vector from the origin of the coordinate system to a lattice point of the crystal [13]. Three unit cell vectors are 

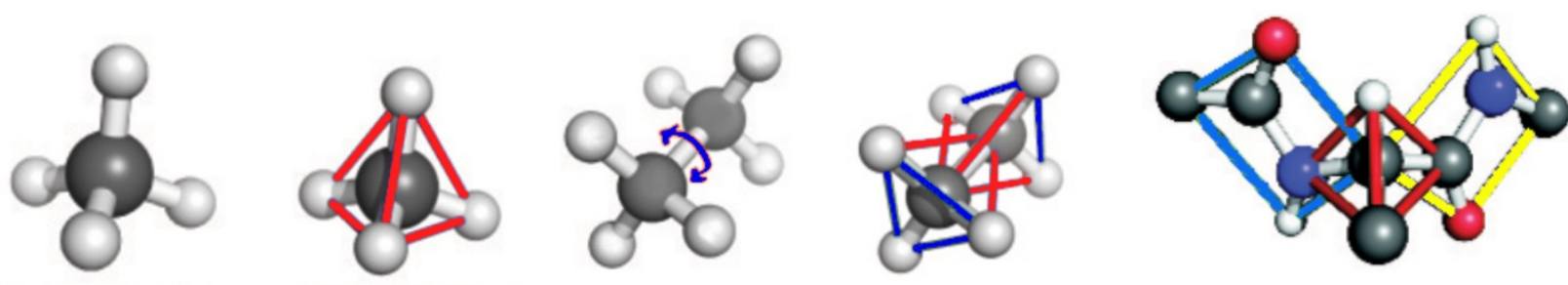

Figure 2 Methane (two left images) is rigid because all pair-wise distances between atoms are fixed. In ethane (center), a carbon atom (gray) and its bonded neighbor atoms form a rigid body. The two bodies share a hinge along the center C-C bond, shown as an abstract bodybar-hinge framework (second from right). A protein's peptide units are modeled as rigid bodies (far right).

needed to describe a unit cell. A symmetry operation is a transformation operation (represented as a matrix) acting on a protein that produces a copy of it, possibly translated and rotated. The space group referred to in the PDB file is a combination of symmetry operations and a lattice specified by the unit cell vectors.

\section{Generating biological units}

In order to generate the biological assembly form of a protein, the asymmetric unit in a PDB file must be rotated, copied, translated, etc. The number of asymmetric units that make up a biological assembly varies from protein to protein. In some case, the asymmetric unit is the biological form, but in others, two, three, or many more asymmetric units, arranged uniquely in relation to each other, form the biological assembly. A transformation matrix in the PDB file details how chain (s) in the asymmetric unit need to be processed to form the biological form of the protein.

For the purposes of rigidity analysis, building just portions of the biological assembly may be useful. For instance, analyzing increasingly larger portions of a biological assembly can provide insight into the evolution of its flexibility as it builds up from its subunit components, or as it decomposes into its smaller subunits. From a computational point of view, generating a PDB file for the biological unit may not always be possible in the PDB format, which is what KINARI currently supports. Indeed, this format can only accommodate up to 99,999 atoms and up to 36 chains, and many large biological units such as viruses easily exceed these limits.

\section{Mathematics of crystallography}

The symmetry operations, lattices, and space groups used to create a crystal from an asymmetric unit have their foundation in mathematical crystallography. There are seven types of symmetry operations, each of which has a specific matrix representation, but only three (rotation, translation, and screw rotation) are allowed in proteins due to chirality; that is, four symmetry operations are not allowed because they would change the handedness of an alpha-helix of a protein [13] (Figure 3).
Matrix representations of two symmetry operations are shown in Figure 4. On the left, a matrix which has only \pm 1 values on the diagonal and zeroes elsewhere would transform the original protein data by $180^{\circ}$ rotations and reflections about one of the three orthogonal axes. The general structure of a transformation matrix using arbitrary angles is shown on the right.

To generate a crystal, three linearly independent translations are required. If the translations are represented by three vectors, $a, b$, and $c$, then all lattice points are generated by linear combinations of the vectors with integer coeffcients [14]. There are 14 types of Bravais lattices, categorized into seven crystal groups: cubic, tetragonal, rhombohedral, orthorhombic, monoclinic, triclinic, and hexagonal.

For the purpose of this paper, a space group is a combination of one of the 14 lattice types and one to three symmetry operations. While there are 230 distinct space groups, proteins only crystallize into only 65 of them, due to chirality. For example, the protein in PDB file $1 \mathrm{ONJ}$ [15] crystallizes into space group P $4_{1} 2_{1} 2$, with eight symmetry operations; thus it will have eight asymmetric units in the unit cell. The $\mathrm{P}$ and initial 4 indicate a primitive tetragonal lattice type. $4_{1}$ indicates a four-fold screw axis: a $90^{\circ}$ rotation, followed by a translation of $1 / 4$ of the $c$ unit cell vector length. 21 indicates a two-fold screw axis: a $180^{\circ}$ rotation along with a translation of $1 / 2$ of the $a$ unit cell vector length. 2 indicates a $180^{\circ}$ rotation.

\section{Results and discussion}

We now present three detailed case studies of the rigidity analysis of biological assemblies, which highlight why it is important to analyze a protein in its functional form as opposed to just its asymmetric unit. Then, we include three case studies of proteins analyzed in crystal form, and identify a significant, small, or no effect in rigidity. Finally, we show a survey of 982 crystal structures of various sizes, generated from 324 protein structure files.

The rigidity analysis of a protein can find a dominant rigid cluster, whose size is substantially larger than any 


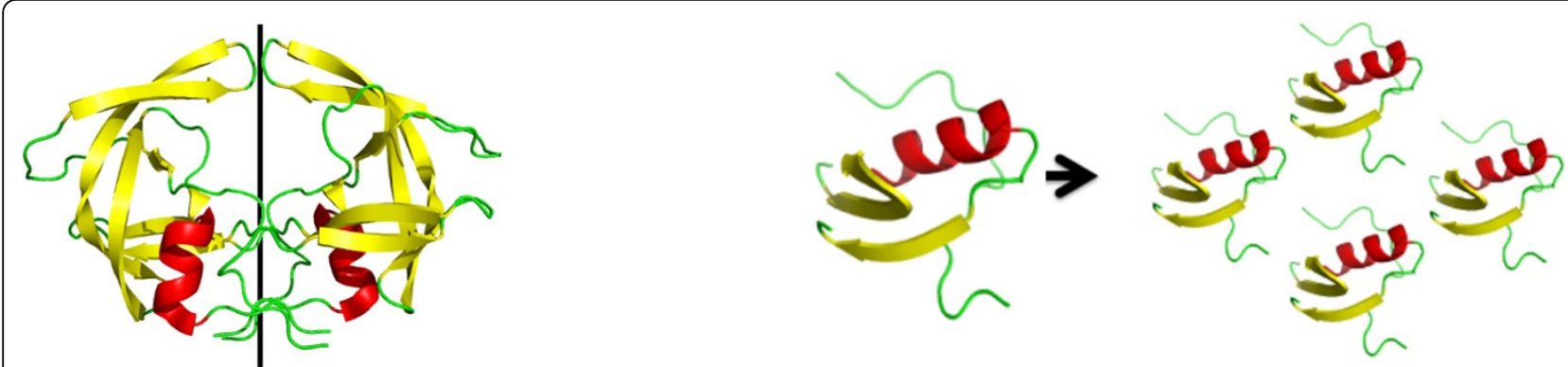

Figure 3 The three symmetry operations allowed in proteins due to chirality are Rotation (left), Translation (right), and Screw Rotation (not shown). These operations do not compromise the handedness of the alpha-helix.

other rigid cluster, or several significant clusters of comparable sizes. Clusters of sizes below a certain threshold are not taken into account in our analysis. We refer to them as insignificant. They typically belong to flexible regions.

Merging of rigid clusters in the biological assembly - PDB structure 1HHP

As a first proof-of-concept step to demonstrate the importance of analyzing the biological assembly versus just a protein's asymmetric unit, we analyzed PDB structure 1HHP. It is the monomeric unit (one-half) of the dimer aspartyl protease, which plays a crucial function in the maturation process of HIV-1. The functional form of the protease is made up of two identical chains, each composed of 99 residues. The PDB file 1HHP contains only the asymmetric unit. Using KINARI's BioAssembly, Curation, and Rigidity Analysis tools, we compared the rigidity of the asymmetric unit in $1 \mathrm{HHP}$ and its biological assembly (Figure 5).

From these results, we see that the asymmetric unit of 1 HHP has a dominant rigid cluster of 710 atoms, while all other clusters have 19 or fewer atoms. In the biological assembly of 1HHP, however, the two monomeric chains have a rigid cluster of approximately 1800 atoms, which is more than double the size in the monomeric unit. This demonstrates that chemical interactions between the two monomers affect the protein's rigidity. Moreover, two flap regions are identified in the biological assembly as being flexible, which is consistent with the studies on the protein's function, in which the flaps move to clamp onto the compounds in the active site of the protease [16].

\section{The biological assembly of a nucleoprotein - PDB file 3000}

The Rift Valley fever virus (RVFV) nucleoprotein [17], PDB ID 3OUO, was chosen to highlight how separate domains of a structure contribute differently to the protein's overall rigidity.

The asymmetric unit in this PDB file contains a 2chained dimer and a 1-chained monomer; each chain has 245 residues. The two biological units for this protein are the hexamer generated with three copies of the dimer and the hexamer generated with six copies of the monomer. Each monomeric chain has an extended, $\mathrm{N}$-terminal arm.

We investigated the rigidity of the asymmetric unit of 3OUO, the monomeric unit of chain $\mathrm{A}$, the monomeric unit of chain $B$, the dimer made of one copy each of chain $A$ and chain $B$, the hexamer made of three copies of the A-B dimer, the monomer made of chain $\mathrm{C}$, the dimer made of two copies of chain $C$, and the hexamer made of six copies of chain $C$ (Figure 6). The tabulations of the rigid clusters for these components of the biological assembly show that as the structure becomes larger by a factor of $n$, the number of rigid clusters of a particular size increase by about the same factor. A closer look at Table 1 further suggests that new rigid clusters are introduced when the hexamer is built from three copies of the dimer and when the hexamer is built from six copies of the monomer. In the first biological assembly, we found three

$$
\left[\begin{array}{ccc} 
\pm 1 & 0 & 0 \\
0 & \pm 1 & 0 \\
0 & 0 & \pm 1
\end{array}\right] \quad\left[\begin{array}{ccc}
\cos \theta & -\sin \theta & 0 \\
\sin \theta & \cos \theta & 0 \\
0 & 0 & \pm 1
\end{array}\right]
$$

Figure 4 Matrices used to generate crystal structures from the asymmetric unit can assume one of several forms, based on the specific transformation that is required to reproduce the repeating crystal units. Depending on the combination of integer \pm 1 values on the diagonal (left), these can be either a rotation by $180^{\circ}$ or a reflection. If a symmetry operation uses rotations with angles other than $180^{\circ}$, the matrix has the form shown on the right, with corresponding values of sin and cos substituted. 


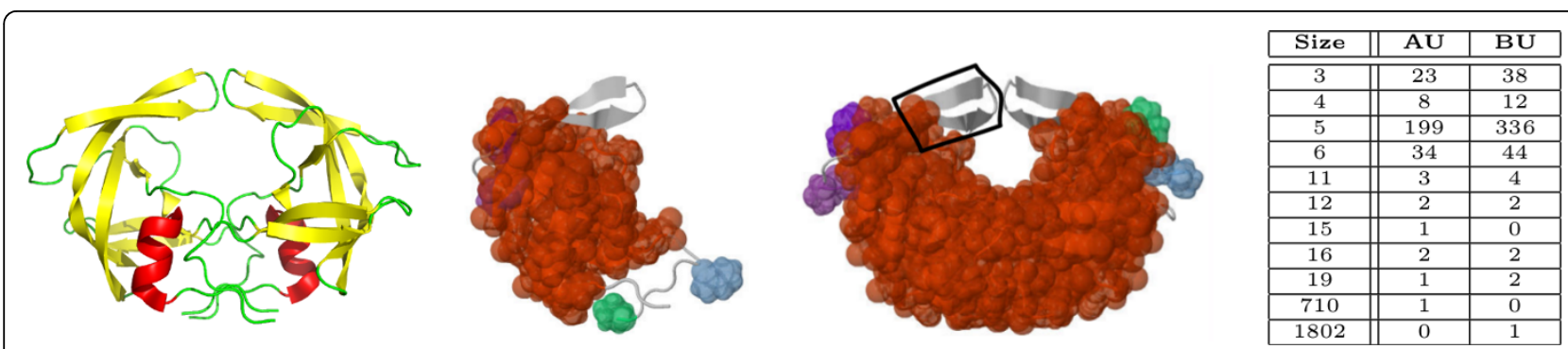

Figure 5 Schematic and rigidity results of HIV-1 Protease. The protein is a dimeric aspartyl protease (PDB file 1HHP)(left). The asymmetric unit (second from lefft) in the PDB file has one significant rigid cluster. Two of the asymmetric units make up the biological form of the protein. When the rigidity of the biological form of the protein is analyzed (second from right), the rigid clusters of the two individual monomers combine into one dominant rigid cluster. The black outlined region designates one of the two $\beta$ hairpin loops often referred to as flaps, which function as chemical scissors and close in on the interior of the protein to facilitate an enzymatic reaction. The number of each type of rigid cluster (right) is listed for the asymmetric and biological units (AU = Asymmetric Unit, BU = Biological Unit).

new clusters with 237 atoms; in the second, we found six new rigid clusters with 118 atoms each. These rigidity results might be explained by the fact that the $\mathrm{N}$-terminal arms bind to a hydrophobic pocket in the surface of the neighboring chain of the biological assembly, which is known to stabilize the hexamer structure [17].
Analyzing how subunits of a scaffolding protein affect the molecule's rigidity - PDB file 3SAQ

The vaccinia virus D13 (PDB file 3SAQ) is a key structural component of the outer scaffold of viral crescents $[18,19]$. The asymmetric unit contains two chains, A and B (Figure 7), with 576 residues each. Two biological

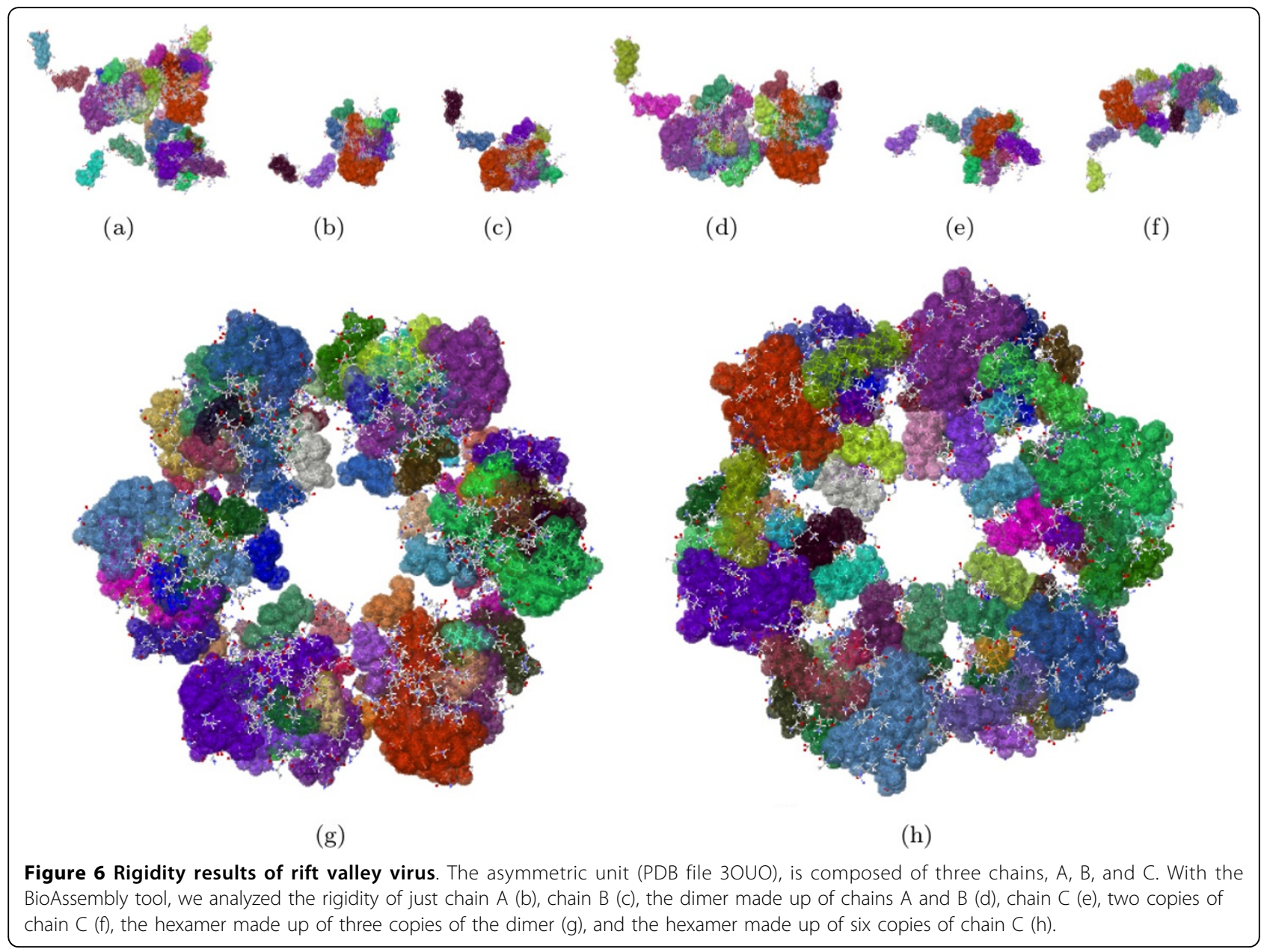


Table 1 Rigidity results for 3000 - the number of each type of rigid cluster is listed for the asymmetric and biological unit $(\mathrm{AU}=$ Asymmetric Unit, $\mathrm{BU}=$ Biological Unit).

\begin{tabular}{|c|c|c|c|c|c|c|c|c|}
\hline Size & $A U$ & BU1a & BU1b & BU1C & BU1 & BU2a & BU2b & BU2 \\
\hline 3 & 215 & 67 & 66 & 142 & 454 & 72 & 148 & 458 \\
\hline 4 & 91 & 33 & 29 & 62 & 186 & 30 & 60 & 182 \\
\hline 5 & 1289 & 429 & 431 & 854 & 2556 & 435 & 854 & 2520 \\
\hline 6 & 159 & 52 & 57 & 107 & 318 & 53 & 102 & 300 \\
\hline 7 & 10 & 3 & 3 & 8 & 27 & 2 & 4 & 12 \\
\hline 11 & 32 & 10 & 10 & 19 & 57 & 13 & 25 & 72 \\
\hline 12 & 31 & 9 & 12 & 20 & 57 & 11 & 21 & 60 \\
\hline 13 & 1 & 1 & 0 & 1 & 3 & 1 & 2 & 6 \\
\hline 15 & 5 & 3 & 2 & 4 & 12 & 1 & 1 & 0 \\
\hline 16 & 11 & 4 & 3 & 7 & 18 & 4 & 9 & 30 \\
\hline 17 & 1 & 0 & 0 & 0 & 0 & 1 & 1 & 0 \\
\hline 19 & 5 & 2 & 1 & 3 & 9 & 2 & 4 & 12 \\
\hline 22 & 6 & 2 & 2 & 4 & 12 & 2 & 4 & 12 \\
\hline 23 & 1 & 0 & 0 & 0 & 0 & 1 & 2 & 6 \\
\hline 30 & 2 & 0 & 2 & 2 & 6 & 0 & 0 & 0 \\
\hline 38 & 1 & 1 & 0 & 1 & 3 & 0 & 0 & 0 \\
\hline 39 & 0 & 0 & 0 & 0 & 0 & 1 & 2 & 6 \\
\hline 55 & 1 & 1 & 0 & 1 & 3 & 0 & 0 & 0 \\
\hline 56 & 2 & 0 & 0 & 0 & 0 & 2 & 4 & 10 \\
\hline 57 & 3 & 1 & 1 & 2 & 6 & 1 & 2 & 6 \\
\hline 58 & 3 & 0 & 1 & 1 & 3 & 1 & 2 & 6 \\
\hline 60 & 0 & 0 & 0 & 0 & 0 & 0 & 0 & 2 \\
\hline 64 & 3 & 1 & 1 & 2 & 6 & 1 & 2 & 6 \\
\hline 66 & 1 & 1 & 0 & 1 & 3 & 0 & 0 & 0 \\
\hline 73 & 1 & 0 & 1 & 1 & 3 & 0 & 0 & 0 \\
\hline 86 & 1 & 0 & 1 & 1 & 0 & 0 & 0 & 0 \\
\hline 89 & 0 & 1 & 0 & 0 & 0 & 0 & 0 & 0 \\
\hline 90 & 0 & 0 & 1 & 0 & 0 & 0 & 0 & 0 \\
\hline 91 & 2 & 1 & 0 & 1 & 3 & 1 & 2 & 6 \\
\hline 92 & 1 & 0 & 0 & 0 & 0 & 2 & 3 & 6 \\
\hline 93 & 2 & 1 & 0 & 1 & 3 & 0 & 0 & 0 \\
\hline 97 & 1 & 0 & 0 & 0 & 0 & 1 & 1 & 0 \\
\hline 100 & 1 & 0 & 1 & 1 & 3 & 0 & 0 & 0 \\
\hline 105 & 1 & 0 & 0 & 0 & 0 & 1 & 2 & 6 \\
\hline 111 & 1 & 0 & 1 & 1 & 6 & 0 & 0 & 0 \\
\hline 113 & 1 & 0 & 1 & 1 & 3 & 0 & 0 & 0 \\
\hline 115 & 1 & 1 & 0 & 1 & 0 & 0 & 0 & 0 \\
\hline 118 & 1 & 1 & 0 & 1 & 0 & 0 & 1 & 6 \\
\hline 122 & 1 & 0 & 0 & 0 & 0 & 1 & 1 & 0 \\
\hline 152 & 0 & 0 & 1 & 0 & 0 & 0 & 0 & 0 \\
\hline 174 & 1 & 2 & 0 & 1 & 3 & 0 & 0 & 0 \\
\hline 175 & 1 & 0 & 0 & 1 & 3 & 0 & 0 & 0 \\
\hline 187 & 1 & 0 & 1 & 1 & 3 & 0 & 0 & 0 \\
\hline 197 & 1 & 0 & 0 & 0 & 0 & 1 & 1 & 0 \\
\hline
\end{tabular}

Table 1 Rigidity results for 3000 - the number of each type of rigid cluster is listed for the asymmetric and biological unit (AU = Asymmetric Unit, BU = Biological Unit). (Continued)

\begin{tabular}{lllllllll}
\hline 221 & 1 & 0 & 0 & 0 & 0 & 1 & 2 & 6 \\
\hline 237 & 0 & 0 & 0 & 0 & 3 & 0 & 0 & 0 \\
\hline 277 & 1 & 0 & 0 & 0 & 0 & 1 & 1 & 0 \\
\hline 381 & 1 & 0 & 0 & 1 & 3 & 0 & 0 & 0 \\
\hline 536 & 1 & 0 & 1 & 1 & 3 & 0 & 0 & 0 \\
\hline 585 & 1 & 1 & 0 & 1 & 3 & 0 & 0 & 0 \\
\hline 737 & 0 & 0 & 0 & 0 & 0 & 0 & 1 & 6
\end{tabular}

Column 2 is the result from analyzing the asymmetric unit, which contains one copy of $A, B$, and $C$ each. Columns 3 and 4 are the results from generating only one-half of the dimer in the asymmetric unit. Columns 5 and 8 are the results from generating the dimer (chains $A$ and $B$ ) and the monomer (chain $C$ ) in the asymmetric unit respectively. Column 7 is the result from generating two copies of the monomer (chain C). And finally, columns 6 and 9 are the results from generating the hexamer; the first from three copies of the dimer (chains $A$ and $\mathrm{B})$, and the second from six copies of the monomer (chain C).

assemblies can be generated from the PDB file. The first one is composed of three copies (subunits) of chain A, and the second is composed of three copies of Chain B.

The rigidity results for the two biological assemblies are surprisingly different, in that assembly 2 , which has a dominant cluster composed of 9475 atoms, is much more rigid than biological assembly 1 , whose significant rigid cluster contains far fewer 2277 atoms. To investigate this, we looked at the chemical interactions among the chains in both biological forms of the protein. Biological assembly 1 has 1092 hydrogen bonds, and 700 hydrophobic interactions, while biological assembly 2 has 1161 hydrogen bonds, and 805 hydrophobic interactions. This suggests that the stabilizing interactions among the three copies of chain B have a stronger effect on the rigidity of biological assembly 2 than do the chemical interactions among the three copies of chain $\mathrm{A}$ in biological assembly 1 . The disparity in rigidity between the two biological assemblies might be explained by findings that multiple copies of both biological units form a honeycomb lattice, which is what provides structural stability for the immature virion membrane [19]. Thus both biological assemblies function cooperatively to perform their structural roles.

In addition, we investigated why the rigidity of the second biological assembly is non-symmetric, even though it is composed of three identical, symmetric, subunits, that are translated and rotated copies of chain B. We compared the hydrogen bonds in each of the three subunits, and found that they had 385,386 , and 384 hydrogen bonds respectively. A further inspection revealed that chain B has several amino acids, for example residue 511, Threonine, to which hydrogen atoms can be assigned in several ways. Threonine is one of two amino acids out of the naturally occurring twenty with two chiral centers 


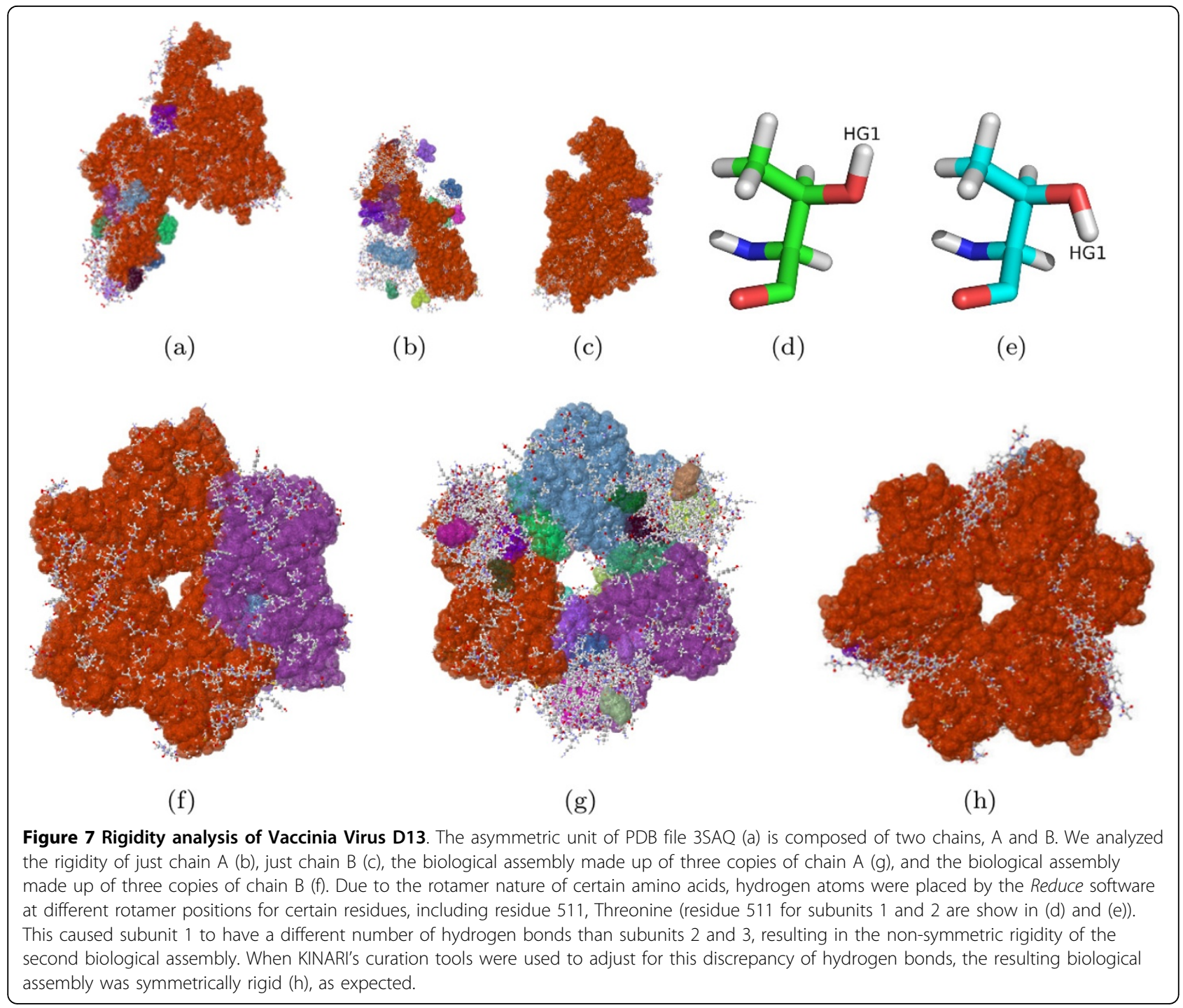

[20], and it can exist as four possible stereoisomers (molecules that have the same molecular formula and sequence of bonded atoms, but that differ only in the three-dimensional orientations of their atoms in space). In addition, Threonine can assume one of several rotamer conformations even among a sample of the same protein $[21,22]$, which explains why the adding of hydrogen atoms to such a residue can be done in one of several ways. The RMSD aligned, superimposed residues 511 for subunit 1 (Figure $7 \mathrm{f}$ ) and subunit 2 (Figure $7 \mathrm{~g}$ ), have their HG1 hydrogen atoms (as were placed using the Reduce software) at different rotamer locations, which explains why one of these engages in a hydrogen bond, and the other does not.

To confirm that indeed the disparity of the number and placement of the hydrogen bonds among the three subunits of the second biological assembly is what causes the protein's non-symmetric rigidity, we performed a pairwise comparison of the stabilizing interactions among the three subunits. We identified where the three subunits differ in their hydrogen bonds. To investigate the effect of adding these hydrogen bonds involving the rotamer residues, we manually added 2, 1 , and 3 hydrogen bonds to the first, second, and third subunits. We used the KINARI curation software (step 4) to insert the hydrogen bonds. In this case, the resulting rigidity of the biological assembly turned out to be symmetric (Figure 7h). Table 2 shows the counts and the sizes of the rigid clusters for the various subunits of PDB structure 3SAQ.

In this case study, it is striking that the placement of such a small number of hydrogen bonds in a subunit of a biological assembly can vastly alter the rigidity of the trimeric protein. In this example, adding 6 hydrogen bonds to an already existing 1161 had a profound 
Table 2 Rigidity results for 3 SAQ - the number of each type of rigid cluster is listed for the asymmetric and biological units ( $A U=$ Asymmetric Unit, $B U=$ Biological Unit).

\begin{tabular}{|c|c|c|c|c|c|}
\hline Size & $A U$ & BU1a & BU1 & BU2a & BU2 \\
\hline 3 & 321 & 303 & 911 & 140 & 434 \\
\hline 4 & 77 & 45 & 132 & 40 & 117 \\
\hline 5 & 1462 & 905 & 2715 & 696 & 2117 \\
\hline 6 & 179 & 187 & 561 & 66 & 217 \\
\hline 7 & 1 & 4 & 12 & 1 & 4 \\
\hline 11 & 16 & 12 & 36 & 8 & 25 \\
\hline 12 & 45 & 31 & 93 & 19 & 56 \\
\hline 13 & 2 & 1 & 3 & 1 & 3 \\
\hline 15 & 2 & 3 & 9 & 0 & 0 \\
\hline 16 & 2 & 1 & 3 & 1 & 3 \\
\hline 19 & 7 & 8 & 24 & 2 & 7 \\
\hline 22 & 1 & 2 & 6 & 0 & 0 \\
\hline 25 & 1 & 2 & 6 & 0 & 0 \\
\hline 33 & 1 & 1 & 3 & 0 & 1 \\
\hline 38 & 1 & 1 & 0 & 0 & 0 \\
\hline 42 & 0 & 0 & 3 & 0 & 0 \\
\hline 48 & 1 & 1 & 3 & 0 & 0 \\
\hline 71 & 1 & 1 & 3 & 0 & 0 \\
\hline 98 & 2 & 1 & 3 & 1 & 3 \\
\hline 104 & 1 & 2 & 6 & 0 & 1 \\
\hline 2277 & 0 & 1 & 3 & 0 & 0 \\
\hline 3912 & 0 & 0 & 0 & 0 & 1 \\
\hline 4562 & 0 & 0 & 0 & 1 & 0 \\
\hline 7883 & 1 & 0 & 0 & 0 & 0 \\
\hline 9475 & 0 & 0 & 0 & 0 & 1 \\
\hline
\end{tabular}

Column 2 is the result from analyzing the asymmetric unit, which contains one copy of A and B each, and columns 3 and 5 are the results from generating only one-third of the biological units listed in columns 4 (three copies of chain A) and 6 (three copies of chain B) respectively.

impact on the rigidity of the biological assembly. This example corroborates with several studies that use rigidity analysis to infer the location of critical residues.

Fox, et al [4], have demonstrated a classification scheme identifying residues that maintain the $3 \mathrm{D}$ shape of a protein. In another study [23], rigidity analysis was performed in which amino acids were in silico mutated to glycine. This is equivalent to removing the hydrogen bond(s) in which the mutated residue engages in. This approach identified residues that could greatly disrupt the protein's rigidity when mutated, and which were conserved among several homologs.

We switch now to analyzing crystal structures.

Dominant cluster aggregation in crystal lattice - PDB file 2YZT

The putative protein from the gram-negative bacterium Thermus thermophilus [24] (PDB file 2YZT), crystallizes in a P $3_{1} 21$ space group, which has 6 associated symmetry operations. Its small size (579 atoms) allows us to quickly analyze the asymmetric unit, unit cell $(1 \times 1 \times 1)$, as well as $2 \times 1 \times 1$ and $2 \times 2 \times 1$ crystal lattices. The unit cell, the $2 \times 1 \times 1$ crystal, and the $2 \times 2 \times 1$ crystal have, respectively, 2, 4, and 8 asymmetric units.

The asymmetric unit is a globular structure with a rigid region and a tail-like segment that remains flexible (Figure 8). The dominant rigid cluster contains 463 atoms, and all other rigid clusters contain fewer than 26 atoms. The unit cell (the protein after the application of the 6 symmetry operations, i.e. the $1 \times 1 \times 1$ crystal) maintains two significant rigid clusters of 463 atoms, but the other rigid clusters of the unit cell combine to form a rigid body containing 2504 atoms, approximately 6 times the size of the significant rigid cluster in the unit cell (Figure 8b). In other words, the significant clusters being adjacent, combine to form the dominant rigid body in the crystal. For the $2 \times 2 \times 1$ crystal, the largest body contains 14,328 atoms (Table 3 and Figure 8c), which is significantly larger than four times the size of the significant rigid cluster in the unit cell. This indicates that the chemical interactions among the unit cells of the crystal have a significant impact on the rigidity of the entire crystal lattice.

The number of significant rigid clusters increase in crystal lattice - PDB File 1UCS

In the previous case study we obtained a very rigid crystal of PDB file 2LZM, with a very large dominant cluster. This is not always the case. Aggregating the asymmetric units of some proteins into a crystal lattice does not appear to greatly effect the rigidity of the resulting crystal structure. We illustrate this with PDB file 1UCS, which contains one of the four types of antifreeze proteins found in marine fish living at sub-zero temperatures [25]. This protein crystallizes in a P $2_{1} 2_{1} 2_{1}$ space group, which has 4 related symmetry operations. The unit cell of 1 UCS is made up of 3 asymmetric units, the $2 \times 1 \times 1$ crystal has 6 asymmetric units, and the $2 \times 2 \times 1$ crystal has 12. In this case, the asymmetric unit does not have a dominant rigid cluster; it has four small significant clusters (Figure 9). Their number increases proportionally to the size of the crystal (Table 4).

\section{Rigidity analysis of Ribonuclease A - PDB files 5RSA and 9RSA}

Our last cast study is on Ribonuclease A, which we analyze based on two PDB files (5RSA and 9RSA). In one case (5RSA), we'll see that aggregating the asymmetric units into a crystal has no bearing on the rigidity of the lattice. 5RSA crystallizes in a P $122_{1} 1$ space group, with only 2 symmetry operations. The asymmetric unit, which contains a single instance of the protein, is made 


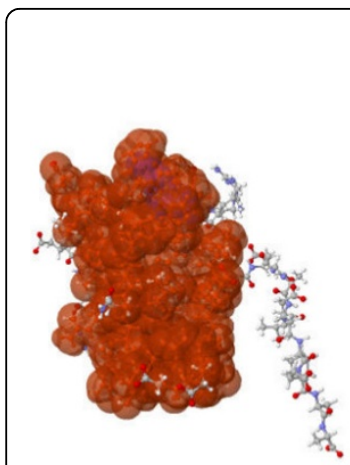

(a) Asymmetric unit.

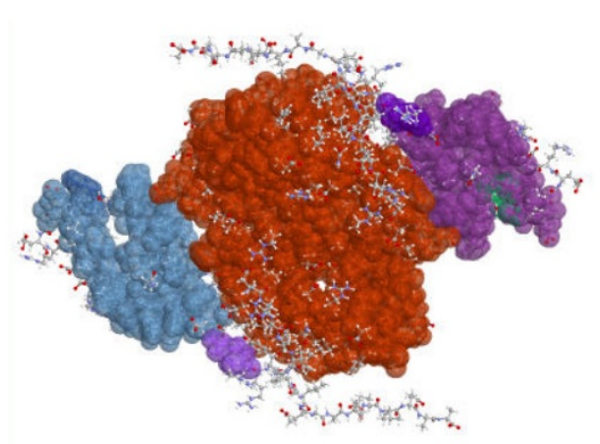

(b) Unit cell: 2 asymmetric units

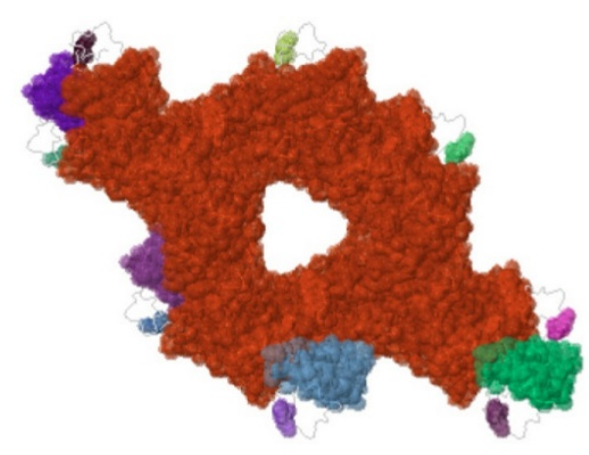

(c) $2 \times 2 \times 1$ Crystal Lattice.

Figure 8 The significant rigid cluster in the asymmetric unit (a) of PDB file 2YZT has $\mathbf{4 6 3}$ atoms. In both the unit cell and the generated lattice, chemical interactions between the neighboring asymmetric units cause the rigid clusters of the individual units to aggregate into a dominant one.

up of 2250 atoms. It has no dominant cluster, and the significant ones are made of approximately 65 atoms. The unit cell and the two crystals we analyzed $(2 \times 1 \times 1$ and $2 \times 2 \times 1)$ all have significant clusters of about the same size (65) (Figure 10). Unlike the previous two case studies (PDB files 1UCS and 2YZT), no clusters (significant or insignificant) are merged at the interface of the units when forming the crystals (Table 5). This may be because no hydrogen bonds form between the two asymmetric units in the unit cell (data not shown). We notice that only 4 new bonds appear between the cells that make up the $2 \times 1 \times 1$ lattice, and only 8 in the $2 \times 2 \times 1$ lattice. However, this small number of bonds does not preclude the formation of larger clusters (see case study for 3SAQ).

This protein is known to retain its function in the crystalline state [5]. This may be due to its maintaining the overall flexibility even in a crystalline form.
RNase A is a widely studied protein, for which there are many structure files in the PDB. One such entry, file 9RSA, is that of a derivative, which is know to exhibit a complete loss of enzymatic activity [26]. We compared the rigidity results of the two forms. PDB structure 9RSA crystallizes in a P $2_{1} 2_{1} 2_{1}$ configuration, and the asymmetric unit contains two instances of the protein. The PDB file 9RSA contains two copies of RNase A. To make the comparison meaningful, we retained only a single instance of RNase A from file 9RSA. Interestingly, the rigidity results of the asymmetric unit of 9RSA has a dominant rigid cluster of 1339 atoms, in stark contrast to the significant rigid clusters in 5RSA.

\section{Survey of 982 crystal structures}

In addition to the previous case studies, we surveyed a dataset of 324 proteins, in 982 biological assembly and

Table 3 Rigidity results for 2 YZT.

\begin{tabular}{|c|c|c|c|c|}
\hline Size & $A U$ & $111.2 \mathrm{YZT}$ & 211.2YZT & $221.2 \mathrm{YZT}$ \\
\hline 3 & 4 & 26 & 49 & 91 \\
\hline 4 & 21 & 106 & 201 & 308 \\
\hline 5 & 122 & 632 & 1165 & 2126 \\
\hline 6 & 22 & 88 & 133 & 1880 \\
\hline 7 & 1 & 4 & 6 & 8 \\
\hline 11 & 5 & 26 & 49 & 93 \\
\hline 12 & 2 & 8 & 12 & 16 \\
\hline 26 & 1 & 4 & 6 & 8 \\
\hline 463 & 1 & 2 & 3 & 4 \\
\hline 2504 & 0 & 1 & 0 & 0 \\
\hline 6084 & 0 & 0 & 1 & 0 \\
\hline 14328 & 0 & 0 & 0 & 1 \\
\hline
\end{tabular}

The number of each type of cluster is shown for the asymmetric unit (AU, column 2), the unit cell (column 3 ), the $2 \times 1 \times 1$ crystal (column 4 ), and $2 \times 2 \times 1$ crystal (column 5) 


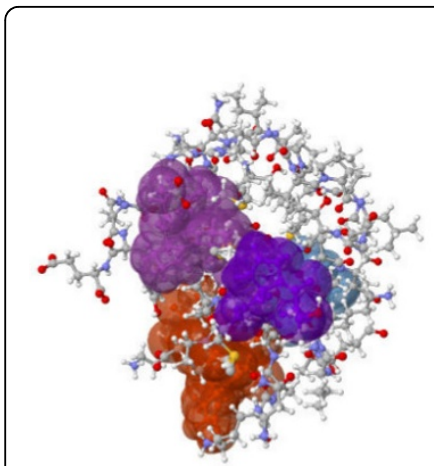

(a) Asymmetric unit

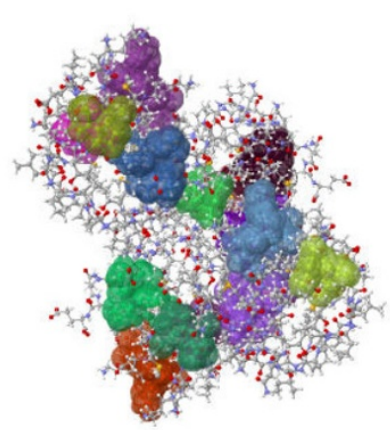

(b) Unit cell of $1 \mathrm{UCS}$

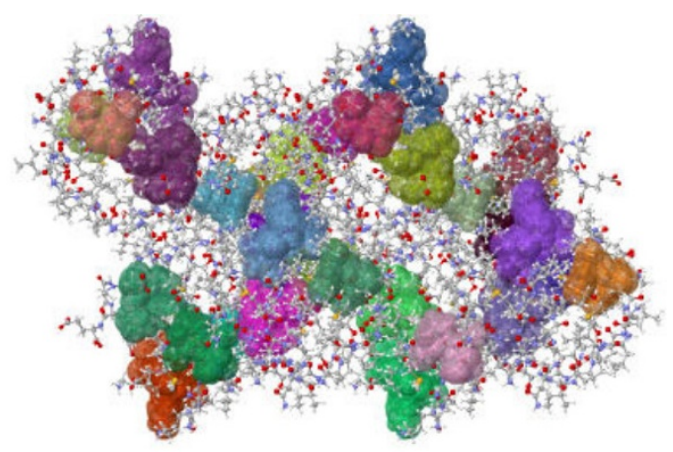

(c) $2 \times 1 \times 1$ Crystal Lattice of 1 UCS

Figure 9 The asymmetric unit (a) of PDB structure 1UCS is composed of four significant rigid clusters (and many more smaller ones that are not displayed). Unlike PDB structure 2YZT (Figure 8a), none of these is substantially larger than the others. In this case, the number of significant rigid clusters in the crystal form is more than the sum of the significant rigid clusters of the asymmetric units. This indicates interactions between the units that affect the rigidity of the crystal structure.

crystal forms. As illustrated in Table 6, it is not biased towards proteins with only large dominant clusters. For each crystal, we tallied what percent of the structure's atoms were in the dominant cluster. We summarized the rigidity of the crystals and information concerning the dominant cluster in three groups, with dominant cluster size larger than $75 \%$, between 50 and $75 \%$, and between 25 and $50 \%$. We also tabulated each crystal's number of hydrogen bonds and hydrophobic interactions. The generated crystals varied in size, the largest having 54,107 atoms (PDB file $3 \mathrm{HON}, 2 \times 2 \times 1$ crystal). Some crystals were made up of as few as a single asymmetric unit, or as many as 48 . The generated crystals varied surprisingly in terms of how many of the structure's atoms were part of the dominant rigid body.
We performed a preliminary classification of the proteins, based on the rigidity of their crystal lattices. We summarize it in Table 7 . For the majority of proteins, we observed a behavior which we call dominant cluster aggregation at all levels (case 1). This means that the crystal contains a dominant cluster of size more than the sum of the dominant clusters in its unit cells or asymmetric units. For 27 of the proteins (case 2), we observed a dominant cluster aggregation at the unit cell level, and no aggregation at the crystal level. Case 3 (33 proteins), shows no change in rigidity among the asymmetric unit and any of the generated crystals. For twenty of the proteins (case 4) the unit cell and $2 \times 2 \times 1$ crystal had rigid bodies that spanned several asymmetric units or unit cells, respectively, but the $2 \times 1 \times 1$ crystal had rigid bodies that were no

Table 4 Rigidity Results for 1UCS: The number of each type of cluster is shown for the asymmetric unit (AU, column 2), the unit cell (column 3 ), the $2 \times 1 \times 1$ crystal (column 4), and the $2 \times 2 \times 1$ crystal (column 5 ).

\begin{tabular}{|c|c|c|c|c|}
\hline Size & $A U$ & 111.1UCS & 211.1UCS & 221.1 UCS \\
\hline 2 & 1 & 5 & 11 & 22 \\
\hline 3 & 63 & 255 & 511 & 1029 \\
\hline 4 & 8 & 32 & 64 & 128 \\
\hline 5 & 181 & 718 & 1434 & 2868 \\
\hline 6 & 46 & 189 & 375 & 747 \\
\hline 7 & 3 & 14 & 30 & 63 \\
\hline 8 & 4 & 16 & 32 & 64 \\
\hline 11 & 1 & 4 & 8 & 16 \\
\hline 12 & 1 & 4 & 8 & 16 \\
\hline 19 & 3 & 12 & 24 & 48 \\
\hline 23 & 1 & 3 & 5 & 10 \\
\hline 27 & 1 & 4 & 8 & 16 \\
\hline 36 & 0 & 1 & 3 & 6 \\
\hline 45 & 1 & 4 & 8 & 16 \\
\hline 67 & 1 & 4 & 8 & 16 \\
\hline
\end{tabular}




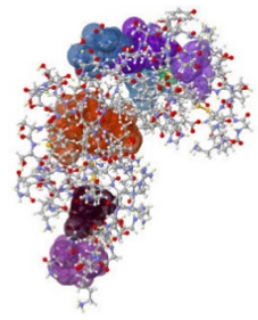

(a) $5 \mathrm{RSA}$

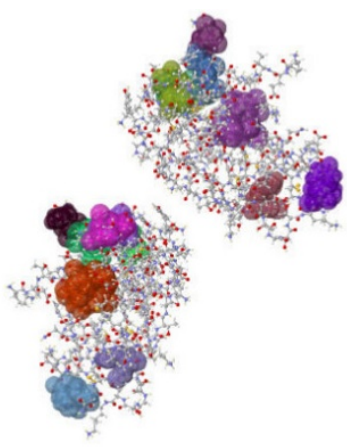

(b) 5RSA unit cell

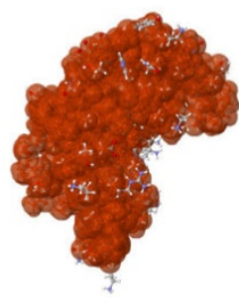

(c) 9RSA

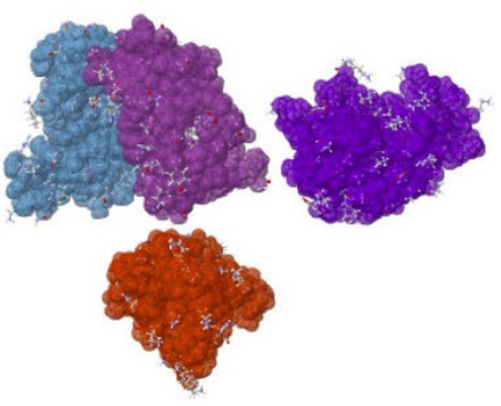

(d) 9RSA unit cell

Figure 10 For Ribonuclease A (PDB file 5RSA), which performs its function even in its crystalline form [5], the asymmetric unit (a) and its unit cell (b) are largely flexible. In contrast, a derivative of the protein, which is known to lose virtually all activity [26], the asymmetric unit (c) and unit cell (d) are both very rigid, and contain far more atoms in the dominant rigid cluster than the structure in file 5RSA.

Table 5 Rigidity results for 5RSA.

\begin{tabular}{|c|c|c|c|c|}
\hline Size & $A U$ & 111.5RSA & 211.5RSA & 221.5RSA \\
\hline 3 & 62 & 124 & 248 & 496 \\
\hline 4 & 20 & 40 & 80 & 160 \\
\hline 5 & 386 & 772 & 1544 & 3088 \\
\hline 6 & 31 & 62 & 124 & 248 \\
\hline 10 & 3 & 6 & 12 & 24 \\
\hline 11 & 3 & 6 & 12 & 24 \\
\hline 12 & 6 & 12 & 24 & 48 \\
\hline 19 & 1 & 2 & 4 & 8 \\
\hline 21 & 1 & 2 & 4 & 8 \\
\hline 22 & 1 & 2 & 4 & 8 \\
\hline 24 & 1 & 2 & 4 & 8 \\
\hline 25 & 2 & 4 & 8 & 16 \\
\hline 29 & 1 & 2 & 4 & 8 \\
\hline 35 & 1 & 2 & 4 & 8 \\
\hline 65 & 1 & 2 & 4 & 8 \\
\hline
\end{tabular}

The number of each type of clusters is shown for the asymmetric unit (AU, column 2), the unit cell (column 3), the $2 \times 1 \times 1$ crystal (column 4 ), and the $2 \times 2 \times 1$ crystal (column 5).

Table 6 Summary of dataset for survey of crystal structures

\begin{tabular}{cc}
\hline & $\mathbf{3 2 4}$ \\
\hline \# Proteins used to generate crystals & 982 \\
\hline Maximum number of asymmetric units in a crystal unit cell & 48 \\
\hline Largest crystal (atoms) & 54,107 \\
\hline \# Crystals with hydrogen bonds between unit cells & 776 \\
\hline \# Structures with 25\% or more but fewer than 50\% of atoms in dominant rigid cluster & $228(18 \%)$ \\
\hline \# Structures with 50\% or more but fewer than 75\% of atoms in dominant rigid cluster & $364(29 \%)$ \\
\hline \# Structures with 75\% or more of atoms in dominant rigid cluster & $334(27 \%)$ \\
\hline
\end{tabular}


Table 7 Preliminary Classification of Proteins According to the Rigidity Properties of Their Crystals

\begin{tabular}{|c|c|c|c|}
\hline & Case & $\begin{array}{c}\text { Num. } \\
\text { Proteins }\end{array}$ & $\begin{array}{c}\% \text { of } \\
\text { Dataset }\end{array}$ \\
\hline 1. & Dominant cluster aggregation at all levels & 192 & 59 \\
\hline 2. & Dominant cluster aggregation at the unit cell level & 27 & 8 \\
\hline 3. & No combining of rigid bodies in unit cell nor in larger crystals & 33 & 10 \\
\hline 4. & Rigid bodies of asymmetric units combined in unit cell and $2 \times 2 \times 1$ crystal, but not for $2 \times 1 \times 1$ crystal & 20 & 6 \\
\hline 5. & $\begin{array}{l}\text { Size of dominant cluster in asymmetric unit and unit cell was the same, but there was aggregation of dominant body } \\
\text { in } 2 \times 1 \times 1 \text { and } 2 \times 2 \times 1 \text { crystals }\end{array}$ & 10 & 4 \\
\hline 6. & Dominant cluster at unit cell that spans multiple asymmetric units but does not aggregate in crystals & 24 & 8 \\
\hline 7. & Other; unclassified. & 18 & 5 \\
\hline
\end{tabular}

bigger than the rigid bodies in the unit cell. This may be because the interactions between the unit cells along one crystal lattice axis may be different. The 10 proteins in case 5 had asymmetric units and unit cells that had the same sized dominant cluster, but for the $2 \times 1 \times 1$ and $2 \times 2 \times 1$ crystals there was a collapse of the dominant cluster. Case 6 contains those proteins with a dominant cluster at the unit cell level that spans multiple asymmetric units but does not aggregate in the larger crystals.

There were some proteins which did not quite fit into any of the six cases. We observed that the asymmetric unit and unit cell of $2 \mathrm{BF} 9$ had no change in rigidity, but when building the $2 \times 1 \times 1$ crystal there was a collapse of rigidity, and the $2 \times 1 \times 1$ and $2 \times 2 \times 1$ crystals had the same rigidity. For the analyses of $6 \mathrm{RXN}$, the asymmetric unit, unit cell, and $2 \times 1 \times 1$ crystal all had no additional collapse, but when the $2 \times 2 \times 1$ crystal was built the size of the largest rigid cluster increased. Although this survey is by no means comprehensive, it already displays patterns of rigidity properties for protein crystals that motivate future extensions of our software for fully understanding protein crystal lattices.

\section{Conclusion}

The rigidity analysis of a single asymmetric unit may not accurately reflect the protein's behavior in the tightly packed crystal environment. Using our KINARI software, we demonstrated that additional functional and rigidity information can be gained by analyzing a protein's biological assembly and/or crystal structure. However, performing a larger scale study would be computationally expensive (due to the size of the molecules involved). Overcoming this limitation will require novel mathematical and computational extensions to our software.

For the analysis of larger assemblies of asymmetric units, we found that relying on "black box" software has to be taken with a grain of salt. In the case of X-ray resolved structures, the PDB files do not contain hydrogen atoms, and these have to be placed with software (such as Reduce). Conversely, using the KINARI curation feature allows one to formulate and verify hypotheses concerning the molecular model, when the placement of atoms or stabilizing interactions needs to be disambiguated.

In the study of crystal structures, we found that some lattices contain rigid bodies that span multiple unit cells, while in other cases, unit cells of crystals retained the rigidity properties of their asymmetric units, in that the rigid bodies did not combine in the larger (crystal) structure. Finally, some crystals were found to be largely flexible, because the unit cells do not have stabilizing interactions among them. In summary, this work shows that rigidity analysis of protein crystals is feasible and has the potential of correlating to important functionrelated aspects of the protein.

For future work, we plan to focus on very large PDB files. They significantly slow down the computation time in building the molecular model, identifying chemical interactions, and performing rigidity analysis. To address these shortcomings, one might take advantage of the symmetry and periodicity in the biological units and crystals. Doing so might preclude from having to compute the locations of all atoms and interactions among symmetric portions of molecules with large symmetries and periodicity.

\section{Methods}

Our computational setup involves the following: we parse the input PDB file, and use the information in it to build the biological unit and desired crystal structure. Then, we apply our KINARI-Web software to place hydrogen atoms, identify chemical interactions, and perform rigidity analysis, which outputs the rigidity clusters. To perform the experiments, we selected a dataset based primarily on protein size, for reasons having to do with the limitations of the current implementation. However, we emphasize that these experiments demonstrated that our software is able to handle relatively large protein structures.

To create the asymmetric unit and crystal lattices, we applied symmetry operations that were given in REMARK 350 of each structure file on each atom of the asymmetric unit. We built the unit cell with supercell.py [27], a Python script. It retrieves the three unit cell vectors from the CRYST1 line of a PDB file, and generates 


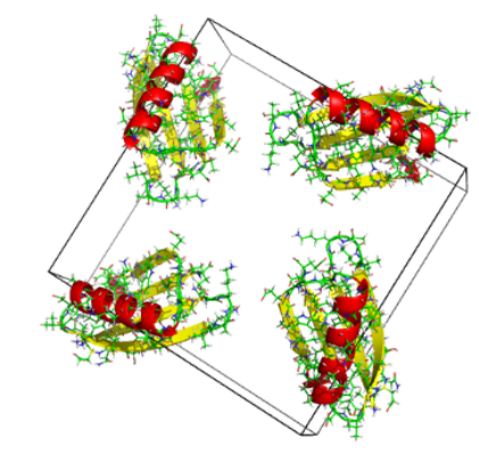

(a) Generated unit cell, colored by secondary structure

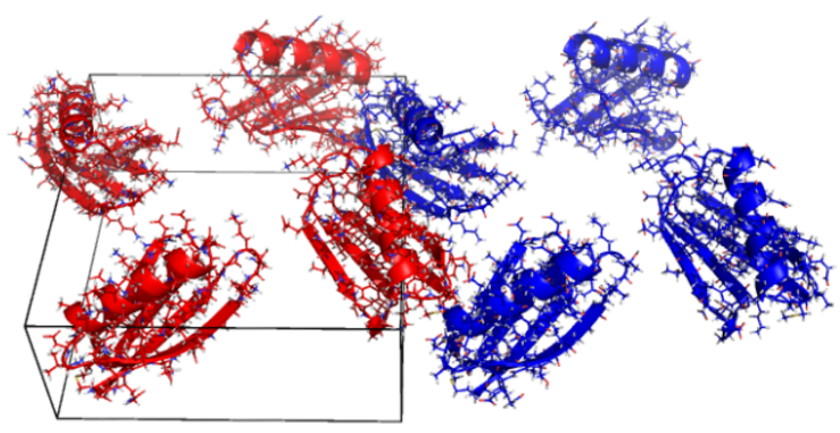

(b) Generated crystal lattice, made up of 2 unit cells (red and blue); bounding box for right unit cell not shown

Figure 11 We generated the unit cell (a) from the asymmetric unit of protein 20N8. In (b), the unit cell is translated using the unit cell vectors to generate a $2 \times 1 \times 1$ crystal lattice.

Table 8 Summary of Experimental Setup

\begin{tabular}{ll}
\hline Step & Description \\
\hline Selection and Curation & 324 PDB files selected for analysis \\
\hline Building of Crystal Lattice & $\begin{array}{l}\text { supercell.py and custom scripts were used to apply symmetry and translation operations on each protein's asymmetric } \\
\text { unit }\end{array}$ \\
\hline $\begin{array}{ll}\text { Performing Rigidity } \\
\text { Analysis }\end{array}$ & The KINARI software was used to calculate the rigid regions of the asymmetric unit and crystal lattices of each protein \\
\hline Identifying Rigid Clusters & The rigidity results were analyzed to determine if interactions of unit cells led to larger rigid clusters. \\
\hline
\end{tabular}

the unit cell by applying the space group symmetry operations on the asymmetric unit. We built the crystal by translating the unit cell in the direction of the three unit cell vectors (Figure 11). For self-checking, we generated the crystal structures using two methods: a custom-built interface to the python script supercell.py [27], and an in-house implementation. An example, illustrating the Immunoglobulin G-binding protein G (PDB file 2ON8) is shown in Figure 1 (left).

Table 8 summarizes the steps of our experimental setup and methods for generating and analyzing crystals build from PDB structure files. The advanced search feature of the PDB was used to select proteins that had between 50 and 150 residues, whose structure was determined using X-Ray crystallography. Only proteins with no DNA nor RNA were selected.

For generating a protein's biological assembly, we used the translation and rotation matrices included in the header of a PDB file. We applied transformation operations that were given as matrices in REMARK 350 of each structure file on each atom of the asymmetric unit. Each matrix contains a $3 \mathrm{D}$ rotation matrix and three translation vectors (one for each axis). The listing of secondary structures also was updated with references to the newly generated atom coordinates.

\section{Competing interests}

The authors declare that they have no competing interests.

\section{Authors' contributions}

IS conceived the research and supervised the project. TL implemented the BioAssembly code, PC and JG the Crystal part, and FJ supervised the code development and performed the KINARI integration. The preliminary experiments, case studies and the survey were done by TL, PC, JG and SM. IS and FJ analyzed the results and wrote the paper.

In this article we present a methodology and experimental computational results that extend our previous work. In the earlier version that appeared in an extended abstract, we reported on rigidity experiments of a limited number of crystal structures. In this work, we introduce and demonstrate KINARI's new feature of generating biological units, and we analyze their rigidity. This article also introduces the crystal and biological unit tools that are freely available on the KINARI website.

\section{Acknowledgements}

Research of FJ and IS was funded by the National Institute of General Medical Sciences DMS-0714934 as part of the Joint program in mathematical biology supported by the Directorate for Mathematical and Physical Sciences of the National Science Foundation and the National Institute of General Medical Sciences of the National Institutes of Health. PC and SM were supported by the NSF-funded Center for Women in Mathematics at Smith College.

\section{Declaration}

Publication of this article was supported by DMS-0714934, NSF CCF-1016988 and DARPA 23 Mathematical Challenges grants of IS.

This article has been published as part of BMC Bioinformatics Volume 14 Supplement 18, 2013: Selected articles from the Second IEEE International Conference on Computational Advances in Bio and Medical Sciences (ICCABS 2012): Bioinformatics. The full contents of the supplement are 
available online at http://www.biomedcentral.com/bmcbioinformatics/ supplements/14/S18.

\section{Relationship with prior work}

This is an expanded publication of the article "Periodic rigidity of protein crystal structures" [28] that was presented at the 2012 IEEE 2nd International Conference on Computational Advances in Biological and Medical Sciences (ICCABS). In this work we perform an analysis of many more crystal structures than were analyzed in the ICCABS publication. Here we also introduce several new features of the KINARI software, including the ability to create biological units from the asymmetric unit in a PDB file. The data for the three case studies of the rigidity analysis of biological assemblies presented in this publication was generated using these new KINARI tools, which are publicly available.

\section{Authors' details}

${ }^{1}$ Department of Mathematics, Smith College, Northampton, MA, USA. ${ }^{2}$ Department of Biological Sciences, Smith College, Northampton, MA, USA. ${ }^{3}$ Department of Computer Science, Central Washington University, Ellensburg, WA, USA. ${ }^{4}$ Department of Computer Science, Smith College, Northampton, MA, USA.

\section{Published: 5 November 2013}

\section{References}

1. Branden C, Tooze J: Introduction to Protein Structure. 2 edition. New York: Garland Publishing, Inc.; 1998.

2. Jacobs D, Rader A, Thorpe M, Kuhn L: Protein Flexibility Predictions using Graph Theory. Proteins 2001, 44(2):150-165.

3. Zhang X, Wozniak J, Matthews B: Protein Flexibility and Adaptability Seen in 25 Crystal Forms of T4 Lysozyme. Journal of Molecular Biology 1995, 250(4):527-552.

4. Fox N, Jagodzinski F, Li Y, Streinu I: KINARI-Web: A Server for Protein Rigidity Analysis. Nucleic Acids Research 2011, 39(Web Server):W177-W183.

5. Bello J, Nowoswiat E: The activity of crystalline ribonuclease A. Biochimica et Biophysica Acta (BBA) - Enzymology and Biological Oxidation 1965, 105(2):325-332.

6. Maxwell J: On the calculation of the equilibrium and stiffness of frames. Philosophical Magazine Series 4 1864, 27:294-299.

7. Tay TS: Rigidity of multigraphs I: linking rigid bodies in $\mathrm{n}$-space. Journal of Combinatorial Theory, Series B 1984, 36:95-112.

8. Jacobs $D$, Hendrickson $B$ : An algorithms for two-dimensional rigidity percolation: the pebble game. Journal of Computational Physics 1997 137:346-365.

9. Lee A, Streinu I: Pebble Game Algorithms and Sparse Graphs. Discrete Mathematics 2008, 308(8):1425-1437.

10. Schlessinger A, Rost B: Protein Flexibility and Rigidity Predicted From Sequence. Proteins 2005, 61:115-126.

11. Rader A, Hespenheide B, Kuhn L, Thorpe M: Protein Unfolding: Rigidity Lost. Proc Natl Acad Sci U S A 2002, 99(6):3540-3545.

12. Del Carpio C, Florea M, Suzuki A, Tsuboi H, Hatakeyama N, Endou A Takaba H, Ichiishi E, Miyamoto A: A graph theoretical approach for assessing bio-macromolecular complex structural stability. Journal of Molecular Modeling 2009, 15:1349-1370.

13. Rupp B: Biomolecular Crystallography: Principles, Practice, and Application to Structural Biology. 1 edition. New York: Garland Science; 2009.

14. Senechal M: Crystalline Symmetries, An Informal Mathematical Introduction Adam Hilger Publishing; 1990.

15. Lou X, Tu X, Pan G, Xu C, Fan R, Lu W, Deng W, Rao P, Teng M, Niu L: Purification, N-terminal sequencing, crystallization and preliminary structural determination of atratoxinb, a short-chain alpha-neurotoxin from Naja atra venom. Acta Crystallogr D Biol Crystallogr 2003, 59(Pt 6):1038-1042.

16. Nicholson LK, Yamazaki T, Torchia DA, Grzesiek S, Bax A, Stahl SJ, Kaufman JD, Wingfield PT, Lam PYS, Jadhav PK: Flexibility and function in HIV-1 protease. Nature Structural Biology 1995, 2(4):274-280.

17. Ferron F, Li Z, Danek El, Luo D, Wong Y, Coutard B, Lantez V, Charrel R, Canard B, Waiz T, Lescar J: The hexamer structure of the Rift Valley fever virus nucleoprotein suggests a mechanism for its assembly into ribonucleoproteiin complexes. PLoS Pathog 2011, 7(5):e1002030.
18. Bahar M, Graham S, Stuart D, Grimes J: Insights into the evolution of a complex virus from the crystal structure of vaccinia virus D13. Structure 2011, 19:1011-1020.

19. Hyun JK, Accurso C, Hijnen M, Schult P, Pettikiriarachchi A, Mitra AK, Coulibaly F: Membrane remodeling by the double-barrel scaffolding protein of poxvirus. PLoS Pathog 2011, 7(9):e1002239.

20. McCoy R, Meyer C, Rose W: Feeding Experiments with Mixtures of Highly Purified Amino Acids. VIII. Isolation and Identification of a New Essential Amino Acid. Journal of Biological Chemistry 1935, 112:283-302.

21. Smith S, Eilers M, Song D, Crocker E, Ying W, Groesbeek M, Aimoto GMMZS Implications of threonine hydrogen bonding in the glycophorin A transmembrane helix dimer. Biophys J 2002, 82:2476-2486.

22. Lovell S, Word J, Richardson J, Richardson D: The penultimate rotamer library. Proteins 2000, 40:389-408.

23. Jagodzinski F, Hardy J, Streinu I: Using Rigidity Anaysis to Probe MutationInduced Structural Changes in Proteins. J Bioinform Comput Biol 2012, 10(3):1242010.

24. Ebihara A, Manzoku M, lino H, Kanagawa M, Shinkai A, Yokoyama S, Kuramitsu S: Crystal structure of uncharacterized protein TTHA1756 from Thermus thermophilus HB8: Structural variety in UPF0150 family proteins. Proteins 2008, 71:2097-2101.

25. Ko TP, Robinson H, Gao YG, Cheng CH, DeVries A, Wang AJ: The refined crystal structure of an eel pout type III antifreeze protein RD1 at 0.62-A resolution reveals structural microheterogeneity of protein and solvation. Biophysical Journal 2003, 84:1228-1237.

26. Nachman J, Miller M, Gilliland G, Carty R, Pincus M, Wlodawer A: Crystal structure of two covalent nucleoside derivatives of ribonuclease $A$. Biochemistry 1990, 29(4):928-937.

27. Holder T: Supercell 2011 [http://www.pymolwiki.org/index.php/Supercell]

28. Clark P, Grant J, Monastra S, Jagodzinski F, Streinu I: Periodic rigidity of protein crystal structures. Computaional Advances in Bio and Medical Sciences (ICCABS), 2012 IEEE 2nd International Conference 2012, 1-6.

29. Wunderlich M, Max E, Roske $Y$, Mueller U, Heinemann U, Schmid F: Optimization of the G $\beta 1$ Domain by Computational Design and by in Vitro Evolution: Structural and Energetic Basis of Stabilization. Journal of Molecular Biology 2007, 373(3):775-784.

\section{doi:10.1186/1471-2105-14-S18-S2}

Cite this article as: Jagodzinski et al:: Rigidity analysis of protein biological assemblies and periodic crystal structures. BMC Bioinformatics 2013 14(Suppl 18):S2.

\section{Submit your next manuscript to BioMed Central and take full advantage of:}

- Convenient online submission

- Thorough peer review

- No space constraints or color figure charges

- Immediate publication on acceptance

- Inclusion in PubMed, CAS, Scopus and Google Scholar

- Research which is freely available for redistribution

Submit your manuscript at www.biomedcentral.com/submit
C Biomed Central 